\title{
Symptoms of gait and coordination impairment in a patient with COVID-19 interstitial pneumonia
}

\author{
Francesca Pistoia ${ }^{1}$ (D) $\cdot$ Raffaele Ornello $^{1} \cdot$ Patrizia Sucapane $^{2} \cdot$ Carmine Marini $^{3} \cdot$ Simona Sacco $^{1}$
}

Received: 16 March 2021 / Accepted: 17 May 2021 / Published online: 22 May 2021

(C) Fondazione Società Italiana di Neurologia 2021

\section{Dear Editor,}

We present a case of gait and coordination impairment developed in a patient with bilateral interstitial pneumonia, history of fever 10 days earlier, and positive detection of COVID-19 IgG.

A 46-year-old male patient was admitted to the emergency department with shortness of breath, difficulties in planning goal-oriented movements, and ataxia: he reported a poor control of purposeful elementary movements, such as moving a comb to the mouth or orienting the remote control for television, associated with intention tremor, truncal, and gait ataxia.

Ten days earlier, he had showed fever in the absence of other symptoms while his mother and sister had presented with cough, hyposmia, and dysgeusia. No neurological diseases or other diseases were reported in the past medical history of the patient with the exception of mild arterial hypertension. No pharmacological treatments were ongoing.

In the emergency department, the patient underwent brain and chest computed tomography: the first did not reveal any pathological findings while the latter showed signs of bilateral interstitial pneumonia (Fig. 1). At admission, vital signs were normal. Blood gas analysis showed low partial pressure of oxygen $(75.3 \mathrm{mmHg})$ : the patient started to be managed with oxygen therapy without the need of a ventilatory support.

Francesca Pistoia

francesca.pistoia@univaq.it

1 Department of Biotechnological and Applied Clinical Sciences, University of L'Aquila, L'Aquila, Italy

2 Department of Neurology, S. Salvatore Hospital, L'Aquila, Italy

3 Department of Internal Medicine, Public Health, Life and Environmental Sciences, University of L'Aquila, L'Aquila, Italy
At the neurological examination, cognitive functions were preserved. No sensory and motor deficits were detected. Deep tendon reflexes in the upper and in the lower limbs were increased with the plantar responses being flexor bilaterally. Cranial nerves were intact. Dysmetria was detected on finger-to-nose and heel-to-shin tests. Static and dynamic balance was significantly impaired, leading to relevant difficulties in mobility. Mild eye nystagmus on horizontal gaze and dysarthria were also detected, together with brief sustained muscle contractions and intention tremor.

Neurological conditions progressively worsened: the postural and action tremor gradually increased thus affecting finemotor movements and resulting in impaired handwriting and disability in daily life activities. Pharmacological management of tremor was almost ineffective.

The patient had two consecutive negative reverse-transcriptase-polymerase-chain-reaction (RT-PCR) findings on the nose and throat swabs. IgM and IgG antibodies were repeatedly analyzed and the patient resulted IgM-negative and IgG-positive. The patient's blood tests showed increased inflammatory markers while autoantibodies and neoplastic markers were absent (Table 1). Cerebrospinal fluid analyses revealed the presence of oligoclonal bands with a mirror pattern but no signs of infection, autoantibodies, or anti-neural antibodies (Table 1). The patient's brain MRI showed a mild leptomeningeal enhancement and EEG were normal.

The patient was treated with oral steroids, antibiotics, and low-molecular-weight heparins for 15 days with full recovery from respiratory symptoms. Rehabilitation was needed for neurological symptoms, which significantly improved in 1 month.

Many neurological manifestations have been described in association to COVID-19 infection: these manifestations include seizures, headache, stroke, Guillain-Barré, and Miller-Fisher syndrome [1]. Encephalopathies, which are apparently linked to COVID-19 infection, seem to share a coordination and gait impairment, denoting a cerebellar syndrome. Poor 


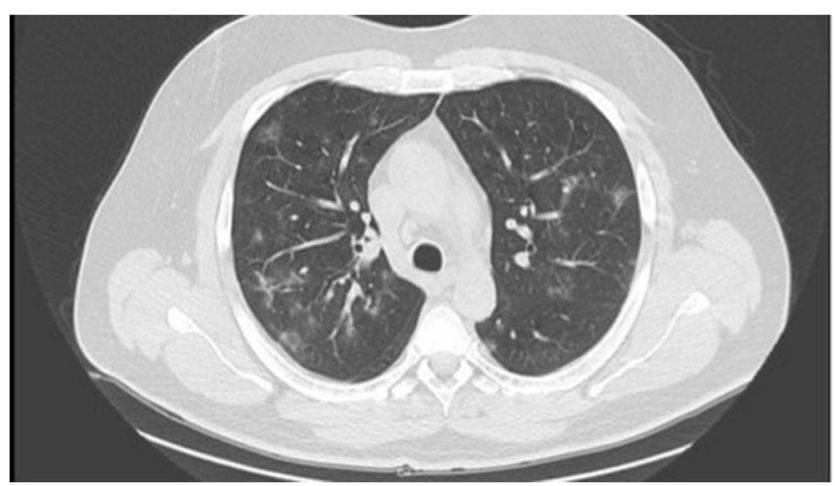

Fig. 1 Thoracic CT scan of the patient coordination and ataxia have been extensively described as rare and treatable post-infectious or para-infectious, immune-mediated phenomena associated with COVID19 [2]. In some cases, opsoclonus and myoclonus have also been described in association with ataxia, denoting a specific condition named opsoclonus-myoclonus-ataxia syndrome (OMAS): this is a rare disorder that is thought to be immune-mediated, with primarily paraneoplastic or para-infectious etiologies [2]. The pathogenetic mechanism leading to neurological syndromes following COVID-19 infection is still controversial. The effects of a proinflammatory cytokine storm, as well

Table 1 Laboratory findings. Figures in bold indicate abnormally elevated values

\begin{tabular}{|c|c|c|}
\hline & Value & Reference range \\
\hline \multicolumn{3}{|l|}{ Blood } \\
\hline White-cell count (per mm³ & 5840 & $4000-10,000$ \\
\hline \multicolumn{3}{|l|}{ Differential count (per $\mathrm{mm}^{3}$ ) } \\
\hline Total neutrophils & 3590 & $2000-6900$ \\
\hline Total lymphocytes & 1430 & $800-3400$ \\
\hline Total monocytes & 660 & $0-900$ \\
\hline Platelet count $\left(* 1000\right.$ per $\left.\mathrm{mm}^{3}\right)$ & 340 & $142-424$ \\
\hline Hemoglobin $(g / l)$ & 15.5 & $13.0-17.0$ \\
\hline \multicolumn{3}{|l|}{ Albumin $(\mathrm{g} / \mathrm{l})$} \\
\hline Alanine aminotransferase (U/l) & 38 & $0-25$ \\
\hline Aspartate aminotransferase (U/1) & 22 & $0-40$ \\
\hline Lactate dehydrogenase (U/1) & 274 & $125-220$ \\
\hline Creatinine $(\mu \mathrm{mol} / \mathrm{l})$ & 0.75 & $0.60-1.20$ \\
\hline Creatine kinase MB (U/l) & 0.5 & $<5.0$ \\
\hline $\operatorname{EGFR}\left(\mathrm{ml} / \mathrm{min} / 1.73 \mathrm{~m}^{2}\right)$ & 110 & $>60$ \\
\hline High-sensitivity cardiac troponin I (pg/ml) & 0.8 & $0-34.2$ \\
\hline Prothrombin time (s) & 14.5 & \\
\hline Activated partial-thromboplastin time (s) & 33 & $25-35$ \\
\hline Fibrinogen $(\mathrm{g} / \mathrm{l})$ & 533 & $200-450$ \\
\hline d-dimer (mg/l) & 0.63 & $0-0.50$ \\
\hline Serum ferritin $(\mu \mathrm{g} / \mathrm{l})$ & 596.6 & $20-280$ \\
\hline Procalcitonin $(\mathrm{ng} / \mathrm{ml})$ & $<0.020$ & $<0.5$ \\
\hline C-reactive protein (mg/dl) & 0.96 & $<0.50$ \\
\hline Anti-nuclear antibodies (ANA) & Absent & \\
\hline Anti-double stranded DNA IgG (IU/ml) & 0 & $<30$ \\
\hline Anti-Sm antibodies (units/ml) & 0 & $0-10$ \\
\hline Anti-RNP antibodies (units/ml) & 0.2 & $0-10$ \\
\hline Anti-Jo1 antibodies (units/ml) & 0 & $0-10$ \\
\hline Anti-SCL70 antibodies (units/ml) & 0 & $0-10$ \\
\hline Anti-SS-A antibodies (units/ml) & 0 & $0-10$ \\
\hline Anti-SS-B antibodies (units/ml) & 0 & $0-10$ \\
\hline Anti-stomach antibodies (APCA) & Present & \\
\hline Anti-cardiolipin IgG (mg/ml) & 3.8 & $0-10$ \\
\hline Anti-cardiolipin IgM (mg/ml) & 2.1 & $0-10$ \\
\hline Anti-myeloperoxidase (pANCA) (units/ml) & 0 & $<20$ \\
\hline Anti-proteinase3 (cANCA) (units/ml) & 0 & $<20$ \\
\hline Anti-citrullinated cyclic peptide antibodies (units/ml) & 1.6 & $<5$ \\
\hline Anti-phospholipid IgG (units/ml) & 2.9 & $<10$ \\
\hline Anti-phospholipid IgM (units/ml) & 3.5 & $<10$ \\
\hline Total IgE (units/ml) & 141 & $<25$ \\
\hline HCV (signal/cutoff) & 0.13 & $<1$ \\
\hline Anti-HBc antibodies (signal/cutoff) & 0.12 & $<1$ \\
\hline HBe antigen (signal/cutoff) & 0.44 & $<1$ \\
\hline $\mathrm{HBsAg}$ (index) & Negative & \\
\hline $\mathrm{HBsAb}$ (units/l) & 0 & $<10$ \\
\hline \multicolumn{3}{|l|}{ Anti-COVID-19 antibodies } \\
\hline $\operatorname{IgG}$ & +++ & \\
\hline
\end{tabular}


Table 1 (continued)

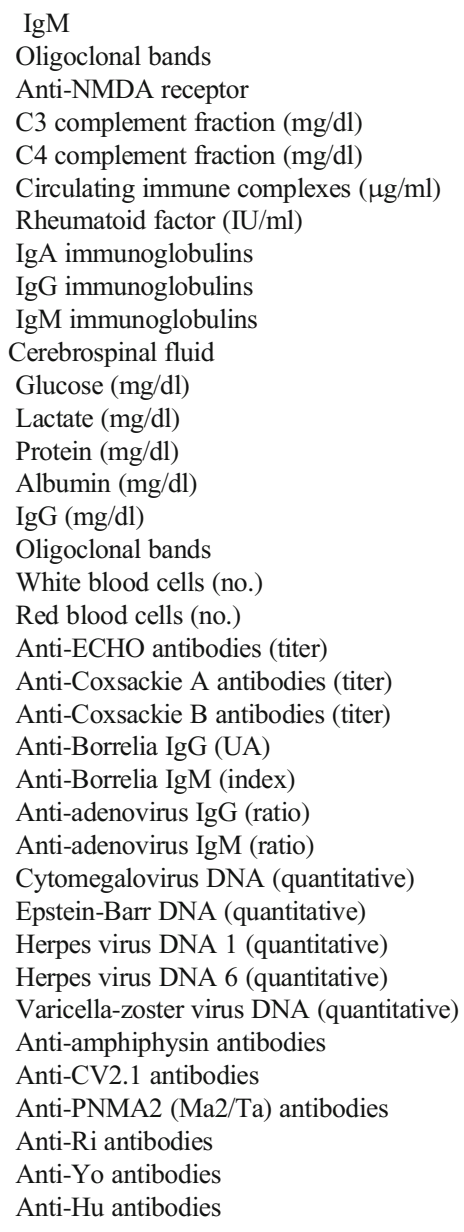

Positive

Negative

$195 \quad 90-180$

$43 \quad 10-40$

$10<<18$

$<14 \quad 0-14$

$213 \quad 70-400$

$1086 \quad 700-1600$

$211 \quad 40-230$

$65 \quad 40-75$

$13.9-10-22$

$22.1 \quad 15-45$

$12.1 \quad 0-35$

$1.46 \quad 0-4$

Positive

$0 \quad 0-5$

$0 \quad 0$

$<1 / 4<1 / 4$

$<1 / 4<1 / 4$

$<1 / 4<1 / 4$

$5.9<10$

$0.8<0.9$

$2.0<1.1$

$0.4<1.1$

Negative

Negative

Negative

Negative

Negative

Absent

Absent

Absent

Absent

Absent

Absent as the secondary damage favored by immune-mediated inflammatory mechanisms, may be involved: the latter are more likely to play a pivotal role in causing neurological dysfunctions when symptoms are delayed with respect to the onset of infection [3]. In our patient, the nose and throat specimens tested negative by rRT-PCR for 2019-nCoV, whereas the serum was tested positive for progressively increasing $\operatorname{IgG}$ levels, which usually start to be detected within 19 days after symptom onset [4]. All other possible causes explaining the observed neurological syndrome were excluded. Moreover, the recognition of intrathecal and mirrored oligoclonal bands suggests an inflammatory central nervous system disorder.

We have to avoid falling into the logical fallacy post hoc, ergo propter hoc, as the appearance of neurological manifestations after the infection does not unequivocally imply a causal relationship between the two conditions. Nevertheless our report endorses the usefulness of serological testing for the identification of neurological patients whose spectrum of symptoms may be suggestive of para-infectious encephalopathy in the COVID era.

\section{Declarations}

Conflict of interest The authors declare no competing interests.

Ethical approval The study was performed in accordance with the ethical standards as laid down in the 1964 Declaration of Helsinki and its later amendments or comparable ethical standards.

Informed consent Although information is anonymized and the submission does not include images that may identify the person, a written informed consent has been obtained from the patient to describe and publish his data about the disease. 


\section{References}

1. Helms J, Kremer S, Merdji H, Clere-Jehl R, Schenck M, Kummerlen C, Collange O, Boulay C, Fafi-Kremer S, Ohana M, Anheim M, Meziani F (2020) Neurologic features in severe SARS-CoV-2 infection. N Engl J Med 382:2268-2270. https://doi.org/10.1056/ NEJMc2008597

2. Chan JL, Murphy KA, Sarna JR (2021) Myoclonus and cerebellar ataxia associated with COVID-19: a case report and systematic review. J Neurol:1-32
3. Fajgenbaum DC, June CH (2020) Cytokine storm. Engl J Med 383: 2255-2273. https://doi.org/10.1056/NEJMra2026131

4. Li G, Chen X, Xu A (2003) Profile of specific antibodies to the SARS-associated coronavirus. N Engl J Med 349:508-509. https:// doi.org/10.1056/NEJM200307313490520

Publisher's note Springer Nature remains neutral with regard to jurisdictional claims in published maps and institutional affiliations. 